Supporting Information for

\title{
A xylochemically-inspired synthesis of lamellarin G trimethyl ether via an enaminone intermediate
}

\author{
Robin Klintworth, ${ }^{a}$ Charles B. de Koning, ${ }^{a}$ Till Opatz ${ }^{\mathrm{b}}$ and Joseph P. Michael ${ }^{*, a}$
}

a. Molecular Sciences Institute, School of Chemistry, University of the Witwatersrand, Wits 2050, Johannesburg, South Africa.

b. Institut für Organische Chemie, Johannes Gutenberg-University, Duesbergweg 10-14, D55128 Mainz, Germany.

\section{NMR SPECTRA}

Page

1. ${ }^{1} \mathrm{H}$ NMR spectrum of $\mathrm{N}$-(3,4-dimethoxyphenethyl)-2-(3,4-dimethoxyphenyl)acetamide (10)......S2

2. ${ }^{1} \mathrm{H}$ NMR spectrum of 3,4-dihydropapaverine hydrochloride $(6 \cdot \mathrm{HCl})$.

3. ${ }^{1} \mathrm{H}$ NMR spectrum of 2-hydroxy-4,5-dimethoxybenzoic acid (12) ................................................S3

4. (a) ${ }^{1}$ H NMR spectrum of methyl 2-hydroxy-4,5-dimethoxybenzoate (13)....................................S3

(b) ${ }^{13} \mathrm{C}$ NMR spectrum of methyl 2-hydroxy-4,5-dimethoxybenzoate (13)....................................S4

5. (a) ${ }^{1} \mathrm{H}$ NMR spectrum of methyl 2-benzyloxy-4,5-dimethoxybenzoate (7)...................................S4

(b) ${ }^{13} \mathrm{C}$ NMR spectrum of methyl 2-benzyloxy-4,5-dimethoxybenzoate (7) ....................................S5

(c) ${ }^{13} \mathrm{C}$ DEPT spectrum of methyl 2-benzyloxy-4,5-dimethoxybenzoate (7)...................................55

6. (a) ${ }^{1}$ H NMR spectrum of (Z)-1-[2-(benzyloxy)-4,5-dimethoxyphenyl]-2-(6,7-dimethoxy-3,4-

dihydroisoquinolin-1(2H)-ylidene)-2-(3,4-dimethoxyphenyl)ethanone (4) . .56

(b) ${ }^{1} \mathrm{H}-{ }^{1} \mathrm{H}$ NOESY spectrum of (Z)-1-[2-(benzyloxy)-4,5-dimethoxyphenyl]-2-(6,7-dimethoxy-3,4-

dihydroisoquinolin-1(2H)-ylidene)-2-(3,4-dimethoxyphenyl)ethanone (4) ........................................S6

(c) ${ }^{13} \mathrm{C}$ NMR spectrum of (Z)-1-[2-(benzyloxy)-4,5-dimethoxyphenyl]-2-(6,7-dimethoxy-3,4-

dihydroisoquinolin-1(2H)-ylidene)-2-(3,4-dimethoxyphenyl)ethanone (4)...................................S7

(d) ${ }^{13} \mathrm{C}$ DEPT spectrum of (Z)-1-[2-(benzyloxy)-4,5-dimethoxyphenyl]-2-(6,7-dimethoxy-3,4-

dihydroisoquinolin-1(2H)-ylidene)-2-(3,4-dimethoxyphenyl)ethanone (4)..................................S7

(e) ${ }^{1} \mathrm{H}-{ }^{13} \mathrm{C} \mathrm{HSQC}$ spectrum of (Z)-1-[2-(benzyloxy)-4,5-dimethoxyphenyl]-2-(6,7-dimethoxy-3,4-

dihydroisoquinolin-1(2H)-ylidene)-2-(3,4-dimethoxyphenyl)ethanone (4)...................................S8

(f) ${ }^{1} \mathrm{H}-{ }^{13} \mathrm{C}$ HMBC spectrum of (Z)-1-[2-(benzyloxy)-4,5-dimethoxyphenyl]-2-(6,7-dimethoxy-3,4-

dihydroisoquinolin-1(2H)-ylidene)-2-(3,4-dimethoxyphenyl)ethanone (4)...................................S8

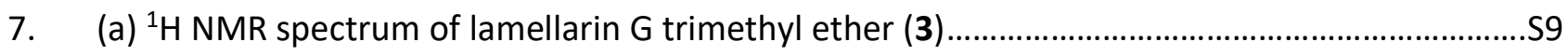

(b) ${ }^{13} \mathrm{C}$ NMR spectrum of lamellarin $\mathrm{G}$ trimethyl ether (3).......................................................... 9 
${ }^{1} \mathrm{H}$ NMR spectrum of $\mathrm{N}$-(3,4-dimethoxyphenethyl)-2-(3,4-dimethoxyphenyl)acetamide (10) (300 $\mathrm{MHz} \mathrm{CDCl}_{3}$ )

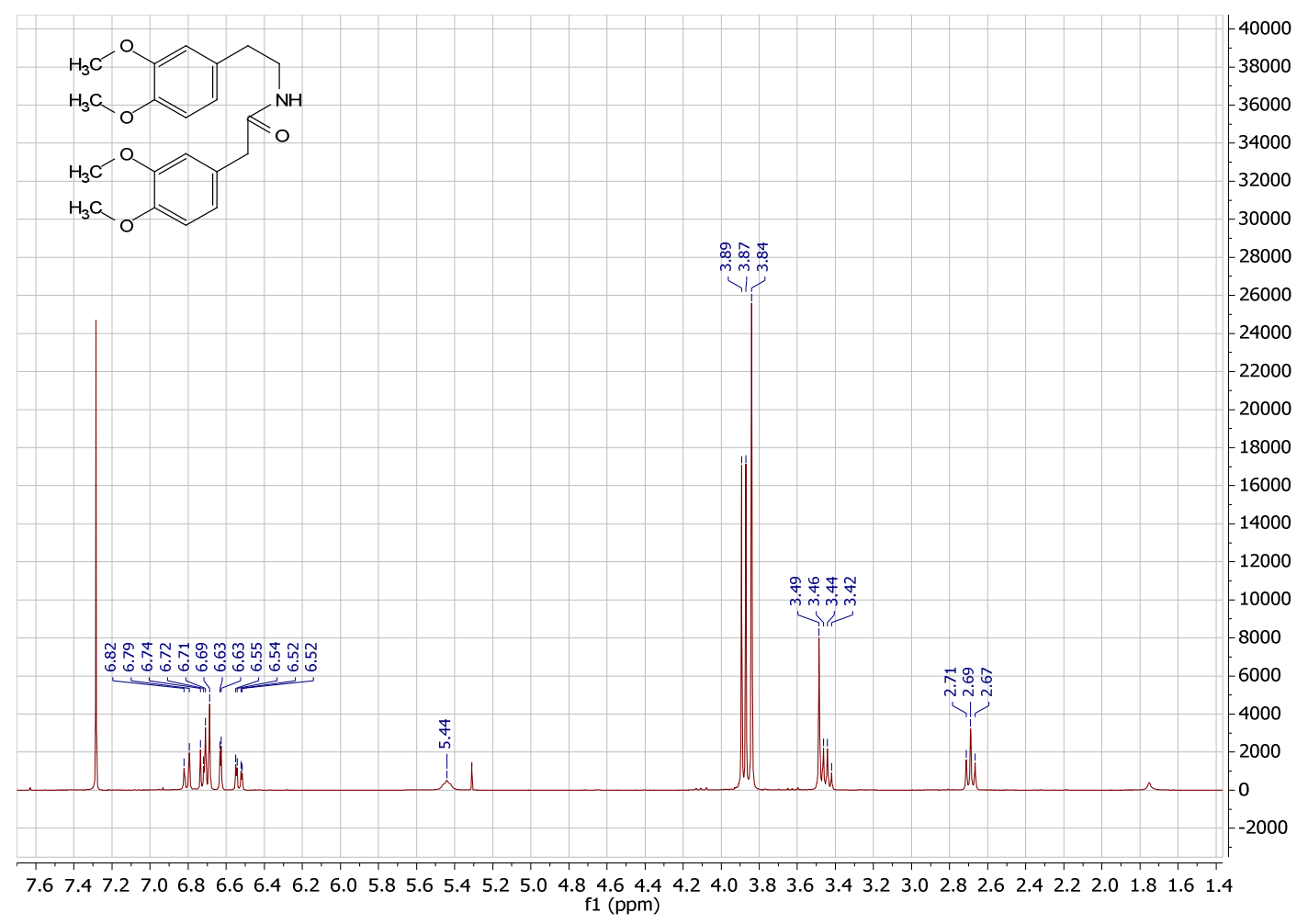

${ }^{1} \mathrm{H}$ NMR spectrum of 3,4-dihydropapaverine hydrochloride $(6 \cdot \mathrm{HCl})\left(300 \mathrm{MHz}, \mathrm{CDCl}_{3}\right)$

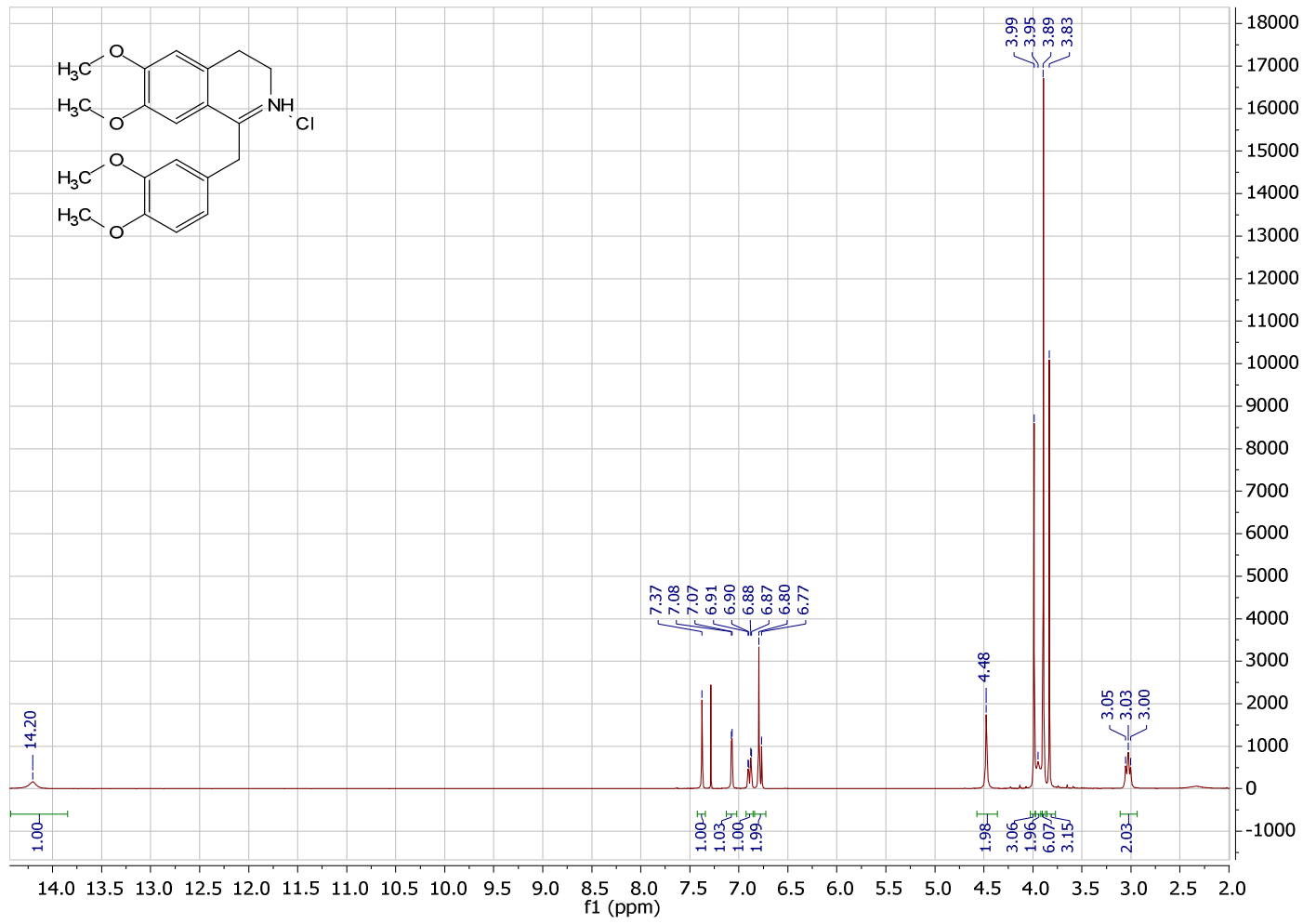


${ }^{1} \mathrm{H}$ NMR spectrum of 2-hydroxy-4,5-dimethoxybenzoic acid (12) (300 MHz, DMSO, $d_{6}$ )

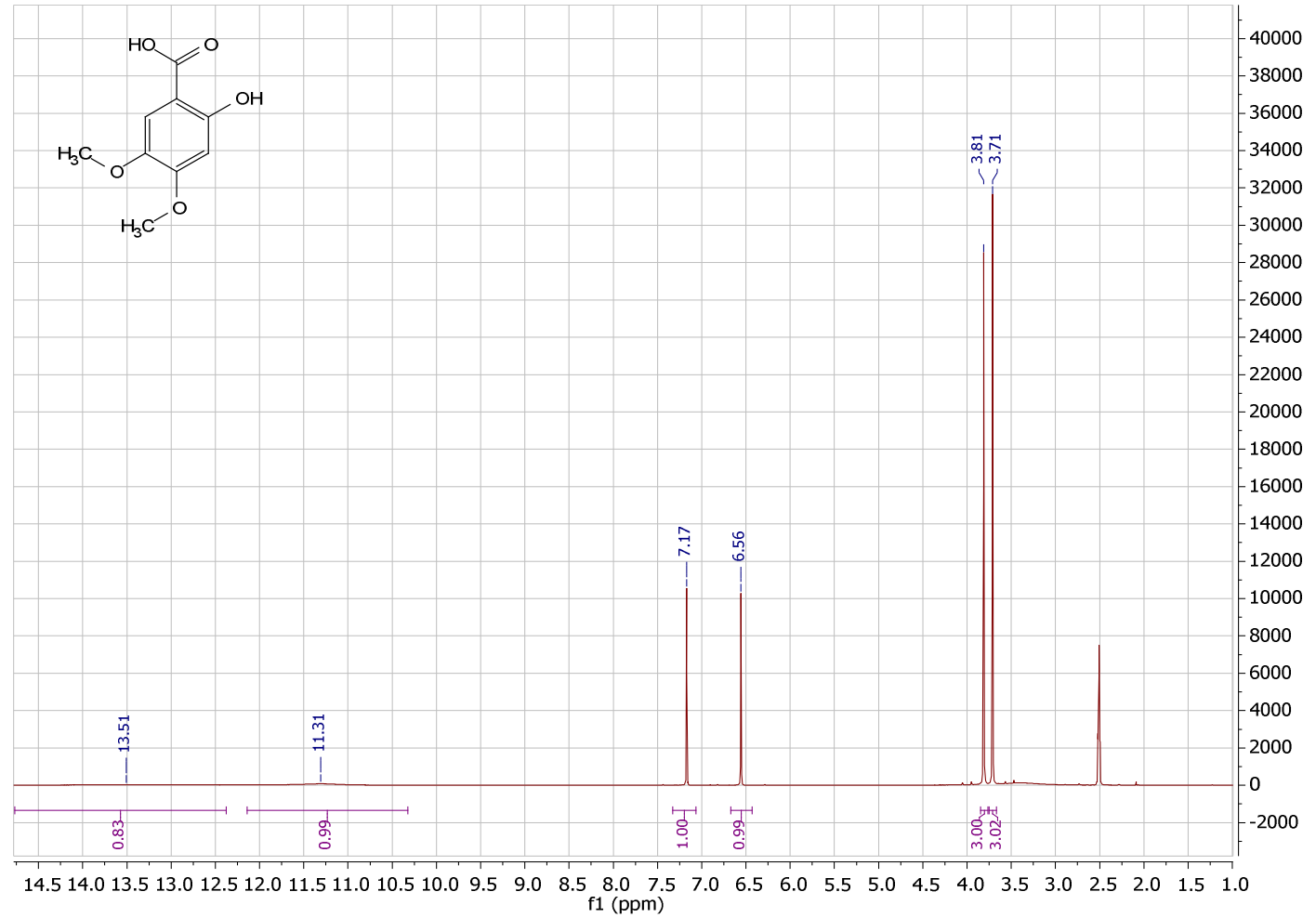

4(a) ${ }^{1} \mathrm{H}$ NMR spectrum of methyl 2-hydroxy-4,5-dimethoxybenzoate (13) (300 $\left.\mathrm{MHz}, \mathrm{CDCl}_{3}\right)$

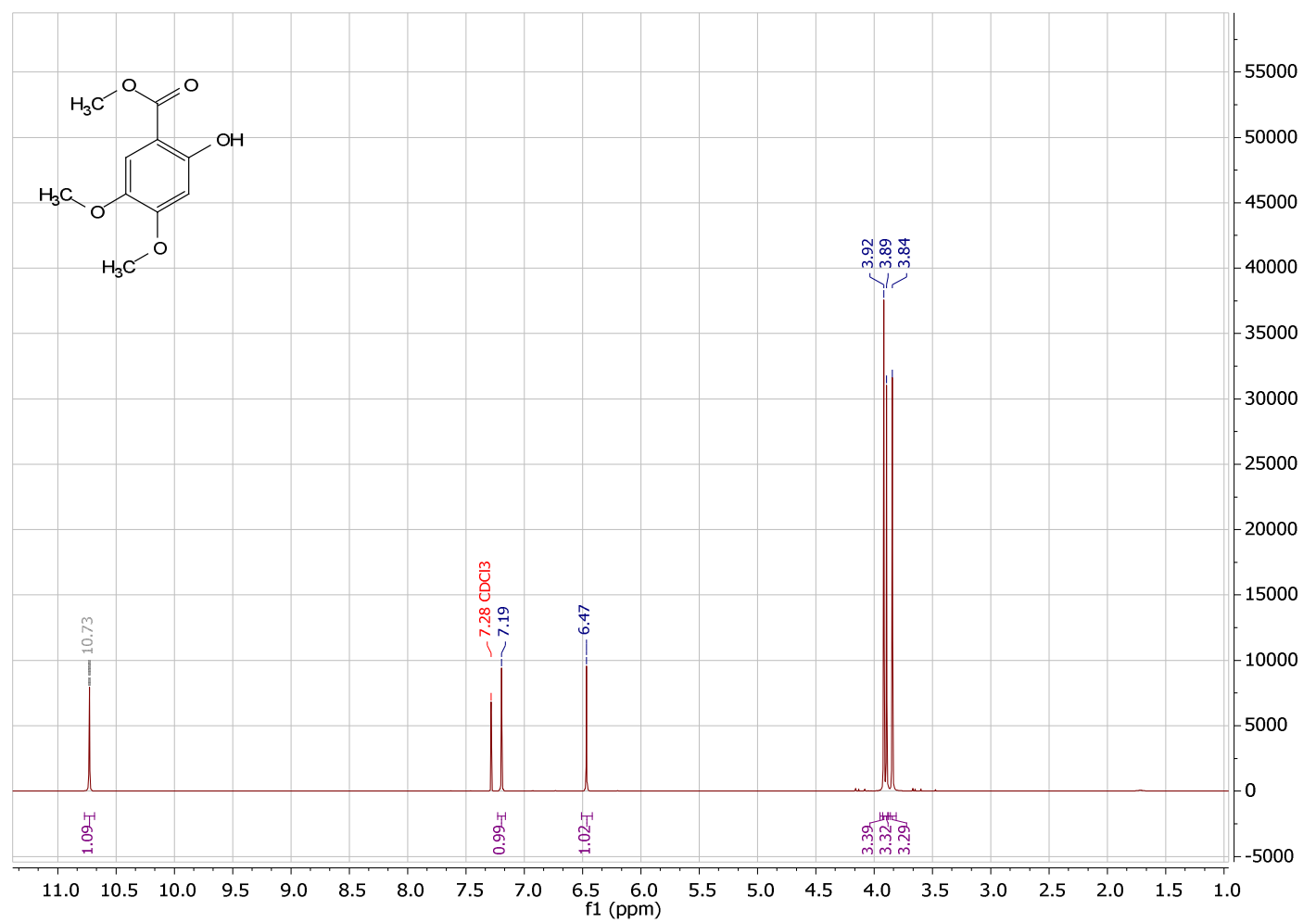


4(b) ${ }^{13} \mathrm{C}\left\{{ }^{1} \mathrm{H}\right\}$ NMR spectrum of methyl 2-hydroxy-4,5-dimethoxybenzoate (13) (75 $\left.\mathrm{MHz}, \mathrm{CDCl}_{3}\right)$

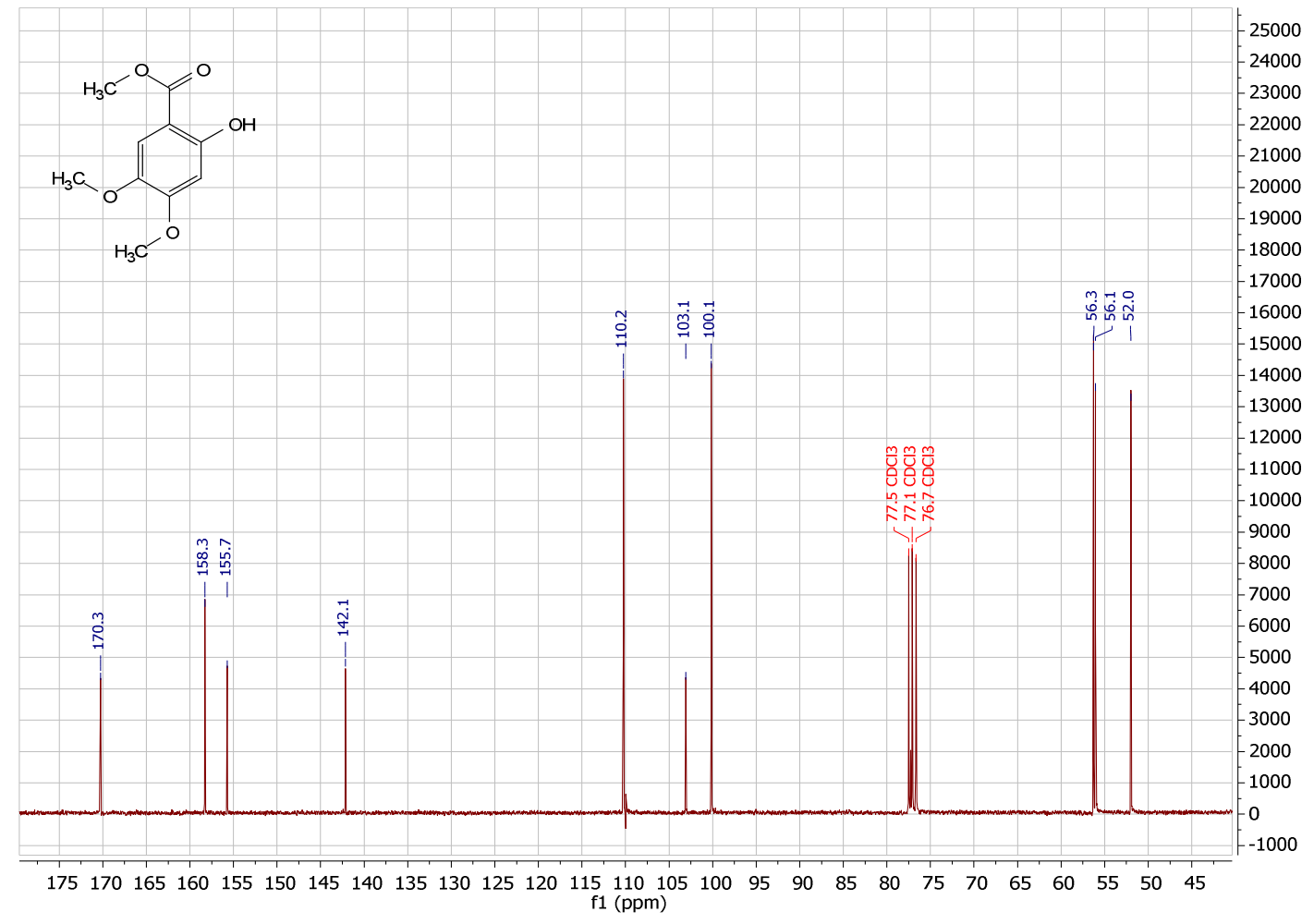

5(a) ${ }^{1} \mathrm{H}$ NMR spectrum of methyl 2-benzyloxy-4,5-dimethoxybenzoate (7) (300 $\mathrm{MHz} \mathrm{CDCl}_{3}$ )

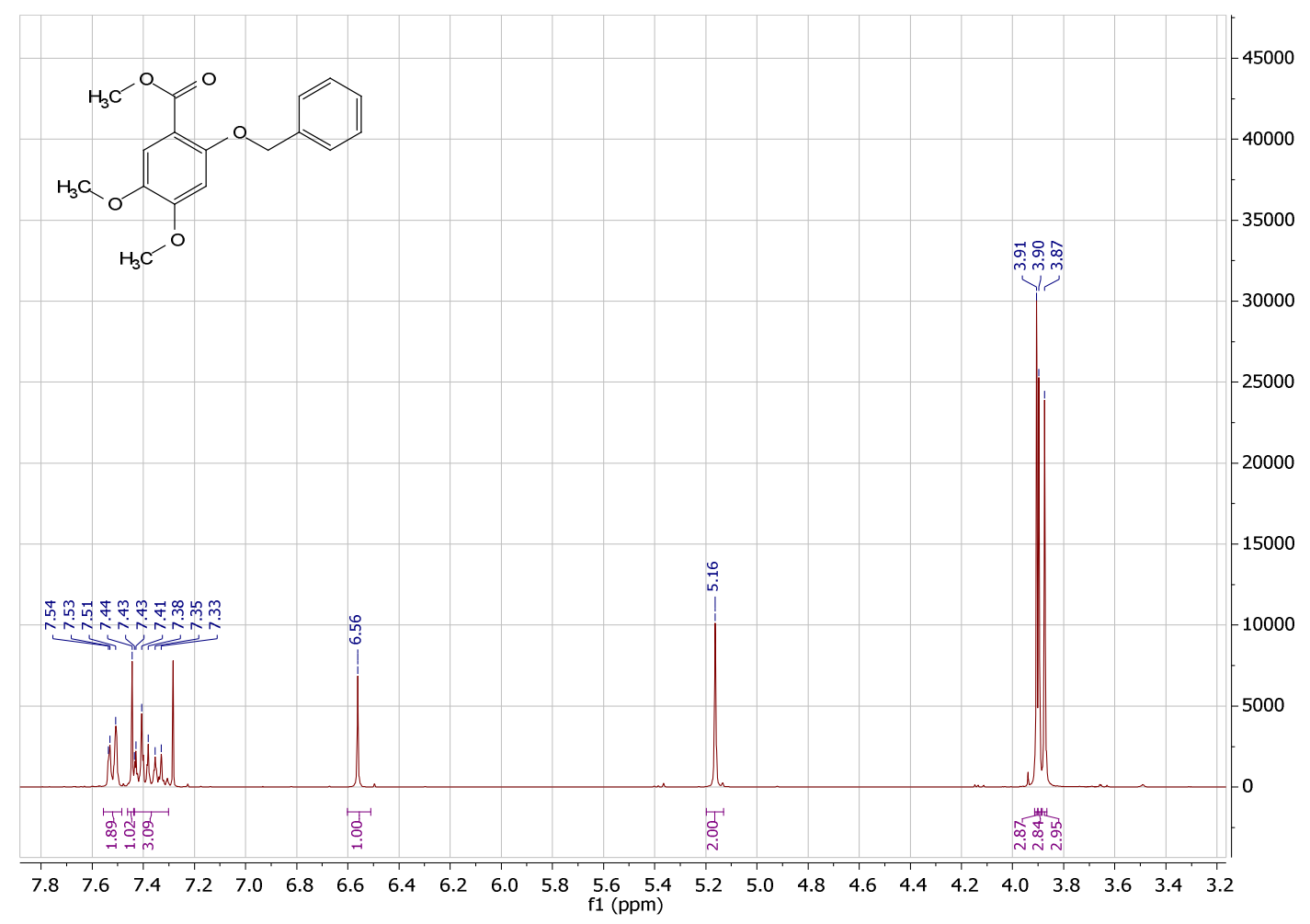


5(b) ${ }^{13} \mathrm{C}\left\{{ }^{1} \mathrm{H}\right\}$ NMR spectrum of methyl 2-benzyloxy-4,5-dimethoxybenzoate (7) (75 $\left.\mathrm{MHz}, \mathrm{CDCl}_{3}\right)$

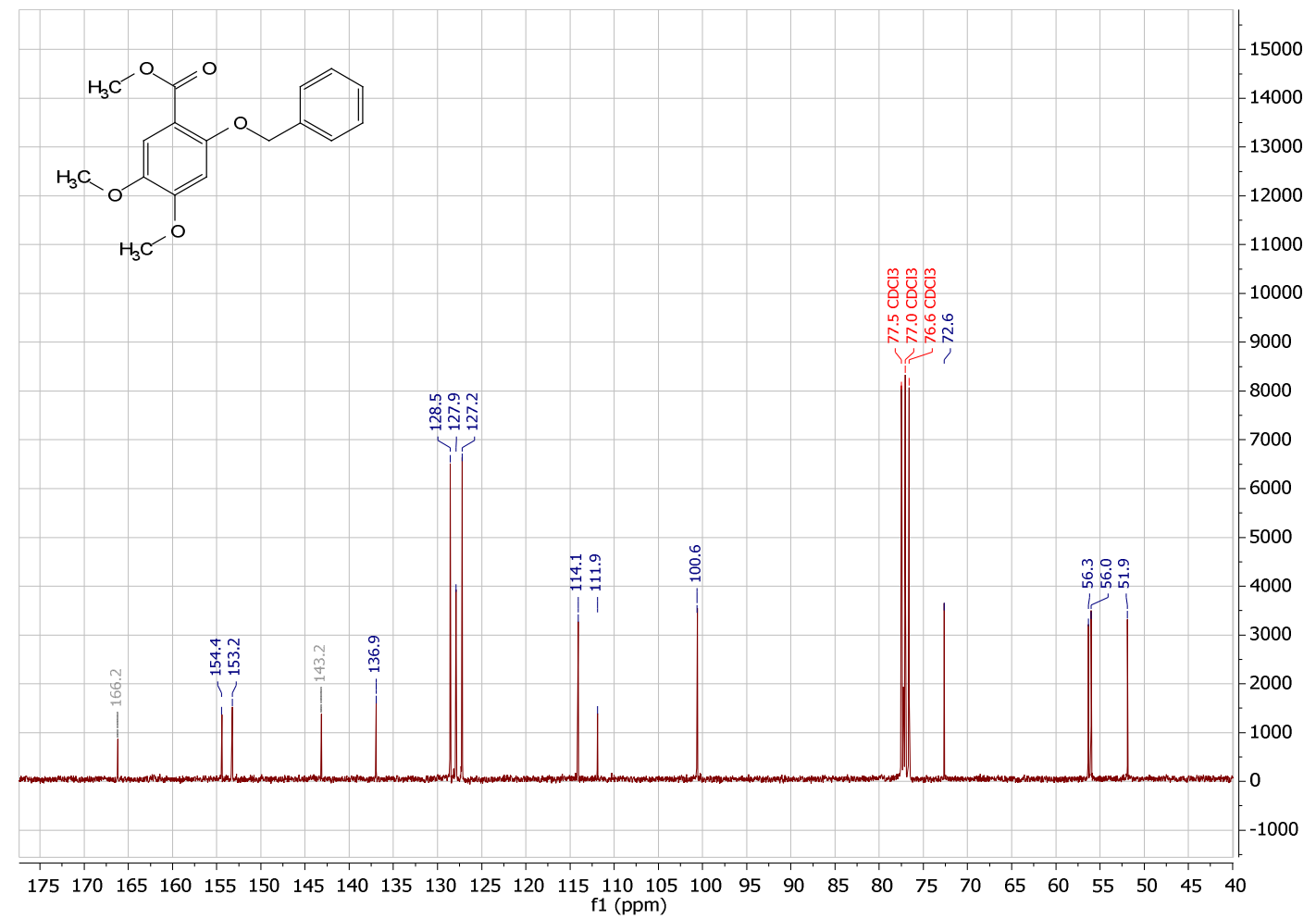

5(c) ${ }^{13} \mathrm{C}$ DEPT spectrum of methyl 2-benzyloxy-4,5-dimethoxybenzoate (7) (75 $\mathrm{MHz}^{\mathrm{CDCl}} \mathrm{Cl}_{3}$

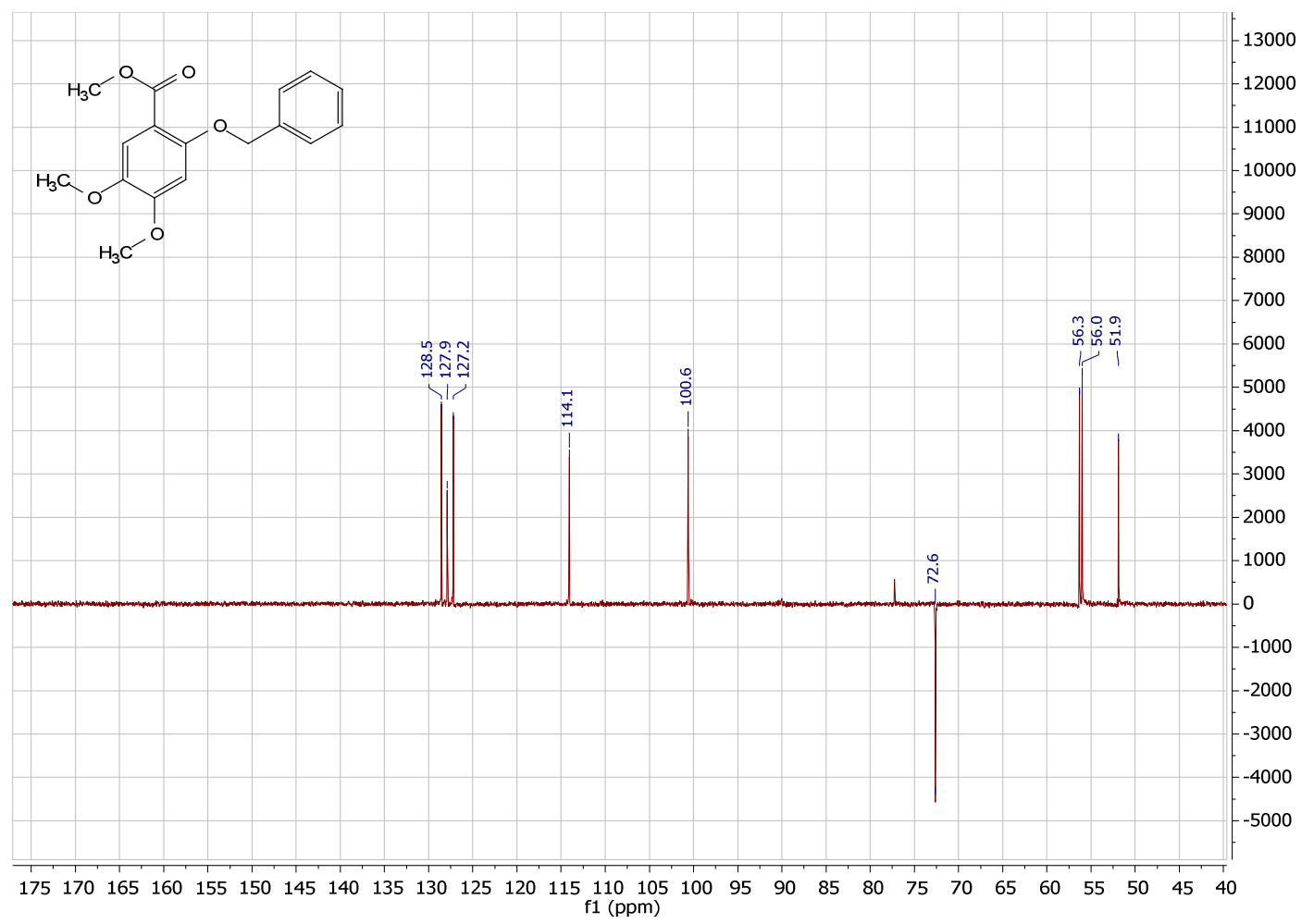


6(a) ${ }^{1}$ H NMR spectrum of (Z)-1-[2-(benzyloxy)-4,5-dimethoxyphenyl]-2-(6,7-dimethoxy-3,4dihydroisoquinolin-1(2H)-ylidene)-2-(3,4-dimethoxyphenyl)ethanone (4) (300 MHz, $\mathrm{CDCl}_{3}$ )

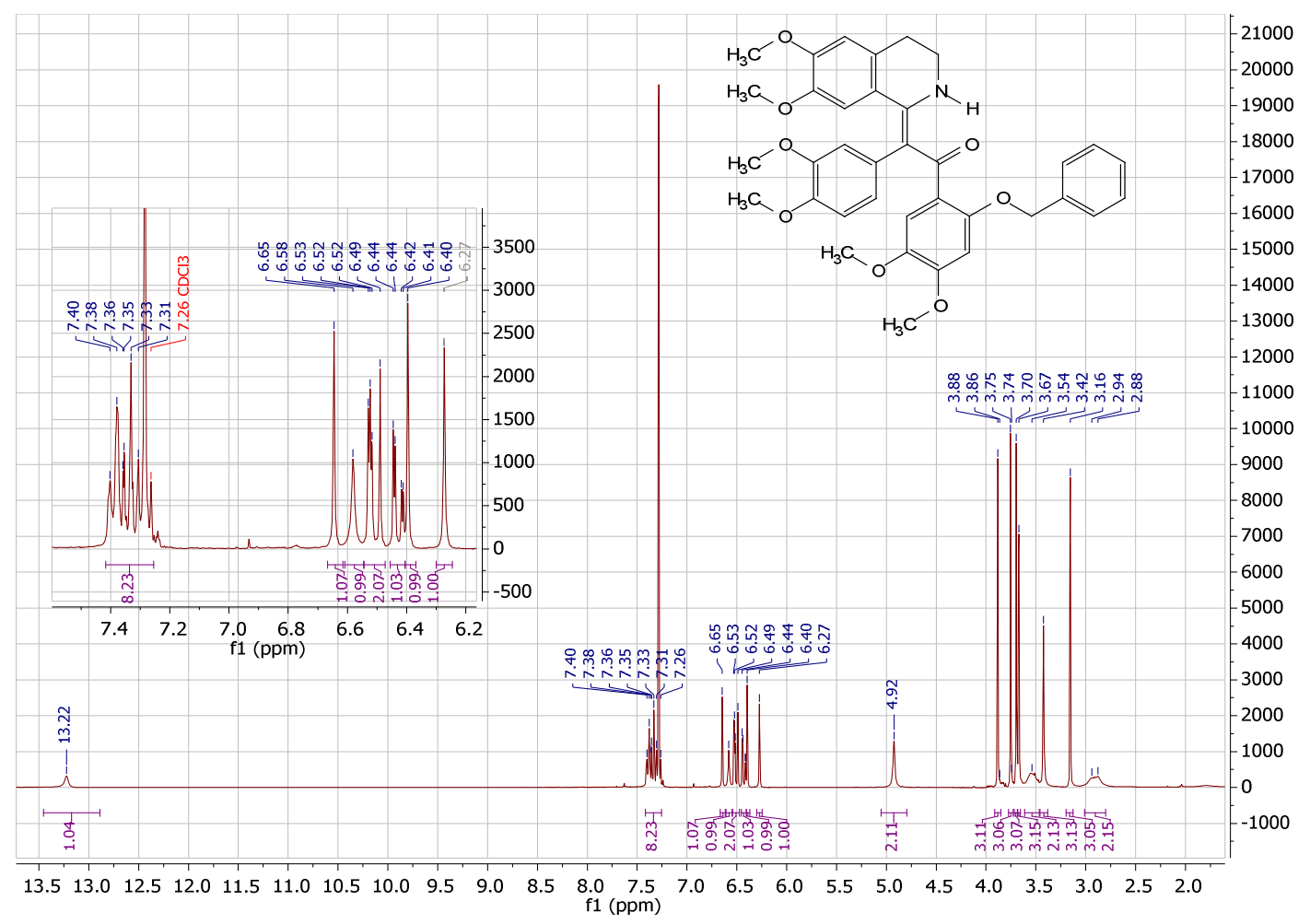

6(b) ${ }^{1} \mathrm{H}-{ }^{1} \mathrm{H}$ NOESY spectrum of (Z)-1-[2-(benzyloxy)-4,5-dimethoxyphenyl]-2-(6,7-dimethoxy-3,4dihydroisoquinolin-1(2H)-ylidene)-2-(3,4-dimethoxyphenyl)ethanone (4) (300 MHz, $\mathrm{CDCl}_{3}$ )

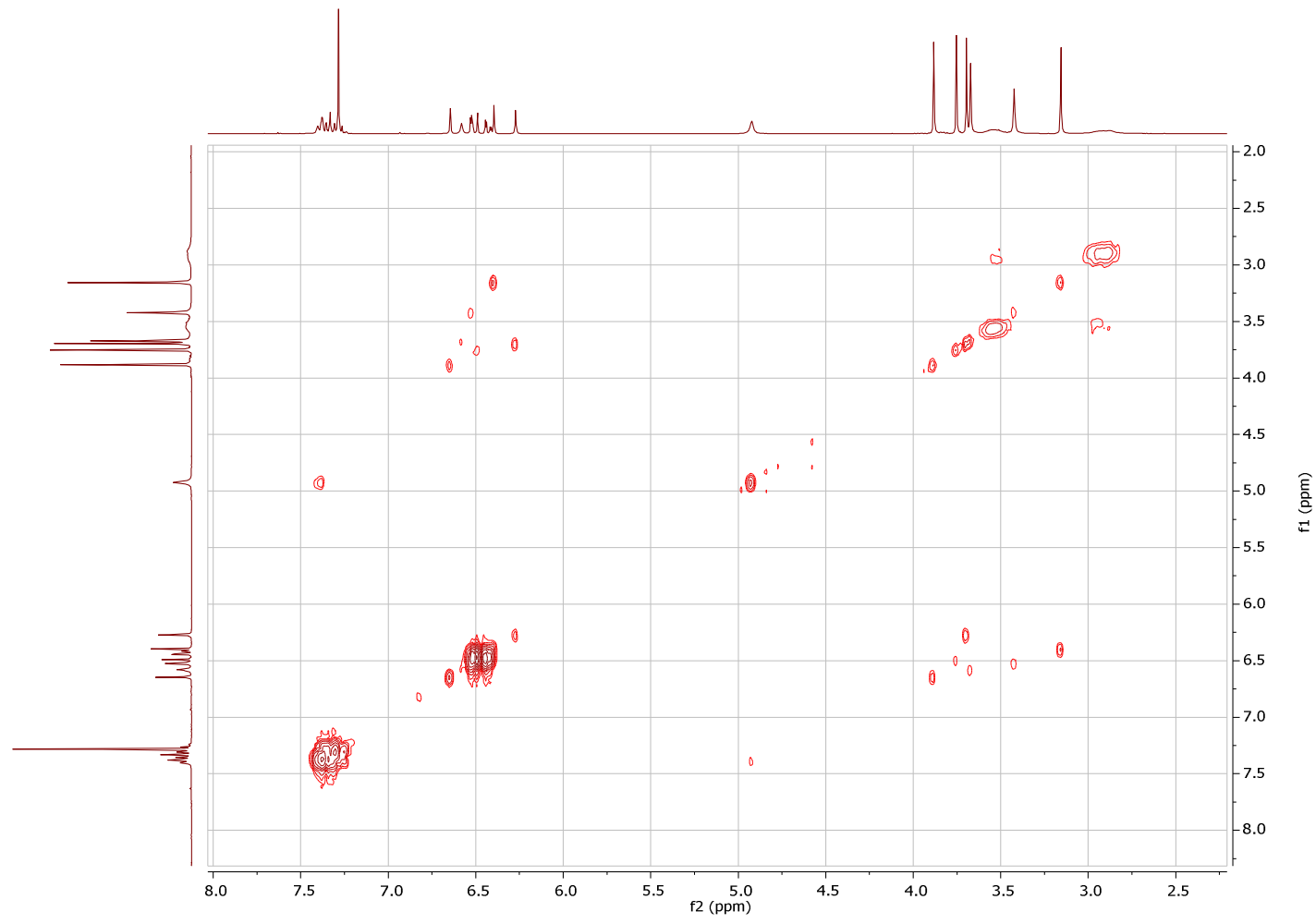


6(c) ${ }^{13} \mathrm{C}\left\{{ }^{1} \mathrm{H}\right\}$ NMR spectrum of (Z)-1-[2-(benzyloxy)-4,5-dimethoxyphenyl]-2-(6,7-dimethoxy-3,4dihydroisoquinolin-1(2H)-ylidene)-2-(3,4-dimethoxyphenyl)ethanone (4) (75 $\mathrm{MHz}, \mathrm{CDCl}_{3}$ )

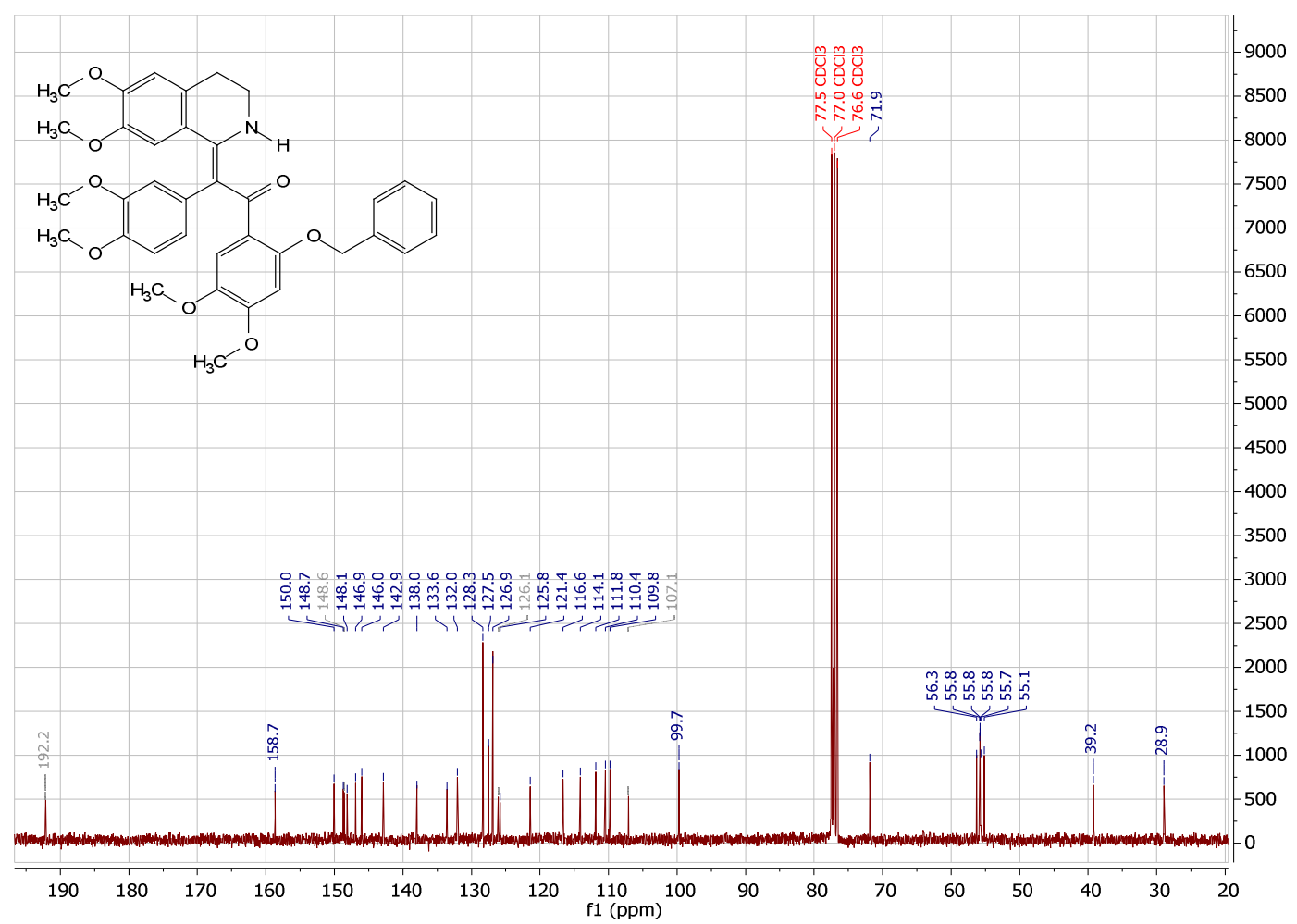

6(d) ${ }^{13} \mathrm{C}$ DEPT spectrum of (Z)-1-[2-(benzyloxy)-4,5-dimethoxyphenyl]-2-(6,7-dimethoxy-3,4dihydroisoquinolin-1(2H)-ylidene)-2-(3,4-dimethoxyphenyl)ethanone (4) (75 $\mathrm{MHz}^{\mathrm{CDCl}} \mathrm{CD}_{3}$

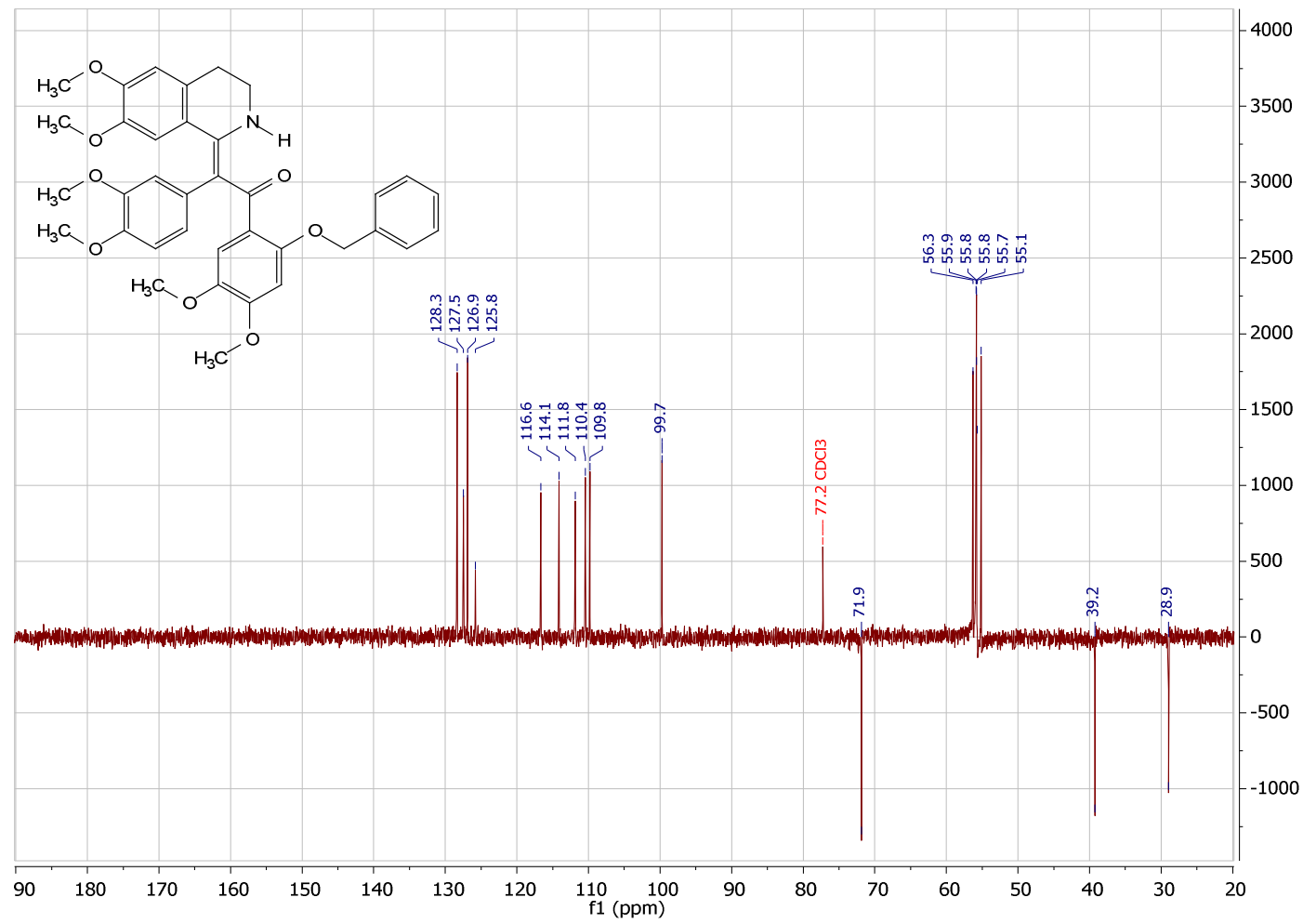


6(e) ${ }^{1} \mathrm{H}-{ }^{13} \mathrm{C}$ HSQC spectrum of (Z)-1-[2-(benzyloxy)-4,5-dimethoxyphenyl]-2-(6,7-dimethoxy-3,4dihydroisoquinolin-1(2H)-ylidene)-2-(3,4-dimethoxyphenyl)ethanone (4) ( $\left.\mathrm{CDCl}_{3}\right)$

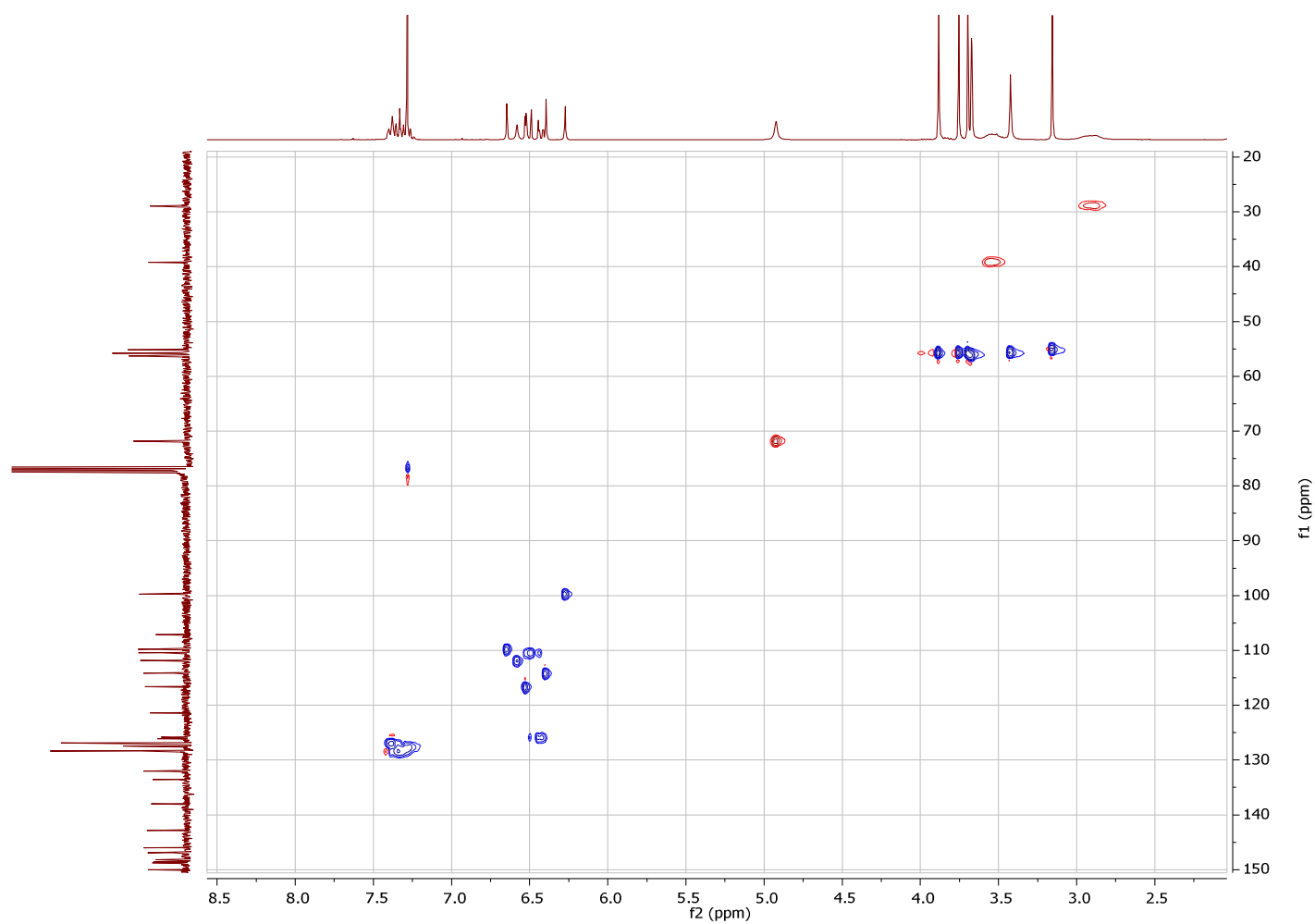

6(f) $\quad{ }^{1} \mathrm{H}-{ }^{13} \mathrm{C}$ HMBC spectrum of (Z)-1-[2-(benzyloxy)-4,5-dimethoxyphenyl]-2-(6,7-dimethoxy-3,4dihydroisoquinolin-1(2H)-ylidene)-2-(3,4-dimethoxyphenyl)ethanone (4) $\left(\mathrm{CDCl}_{3}\right)$

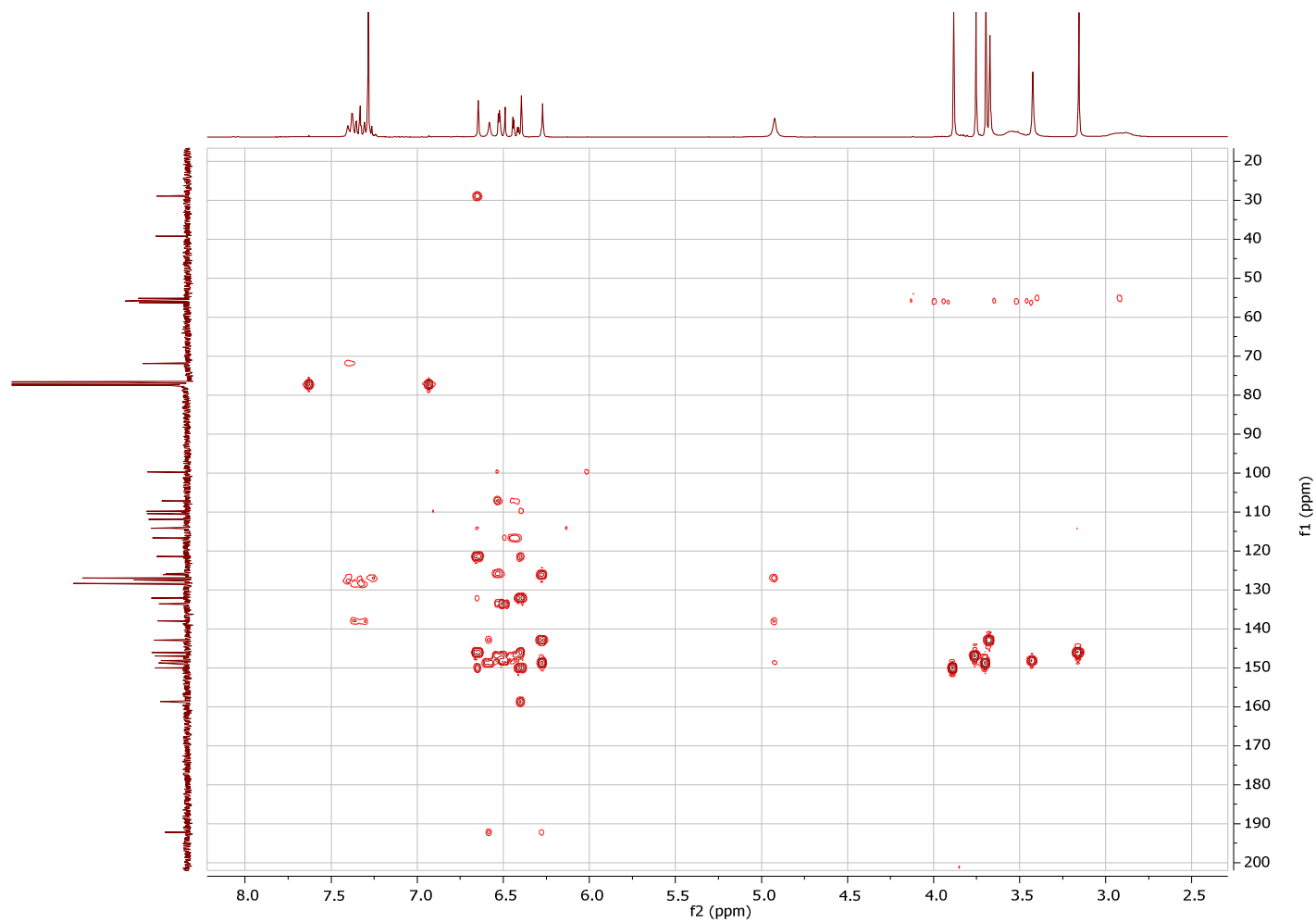


7(a) ${ }^{1} \mathrm{H}$ NMR spectrum of lamellarin G trimethyl ether (3) $\left(300 \mathrm{MHz}, \mathrm{CDCl}_{3}\right)$

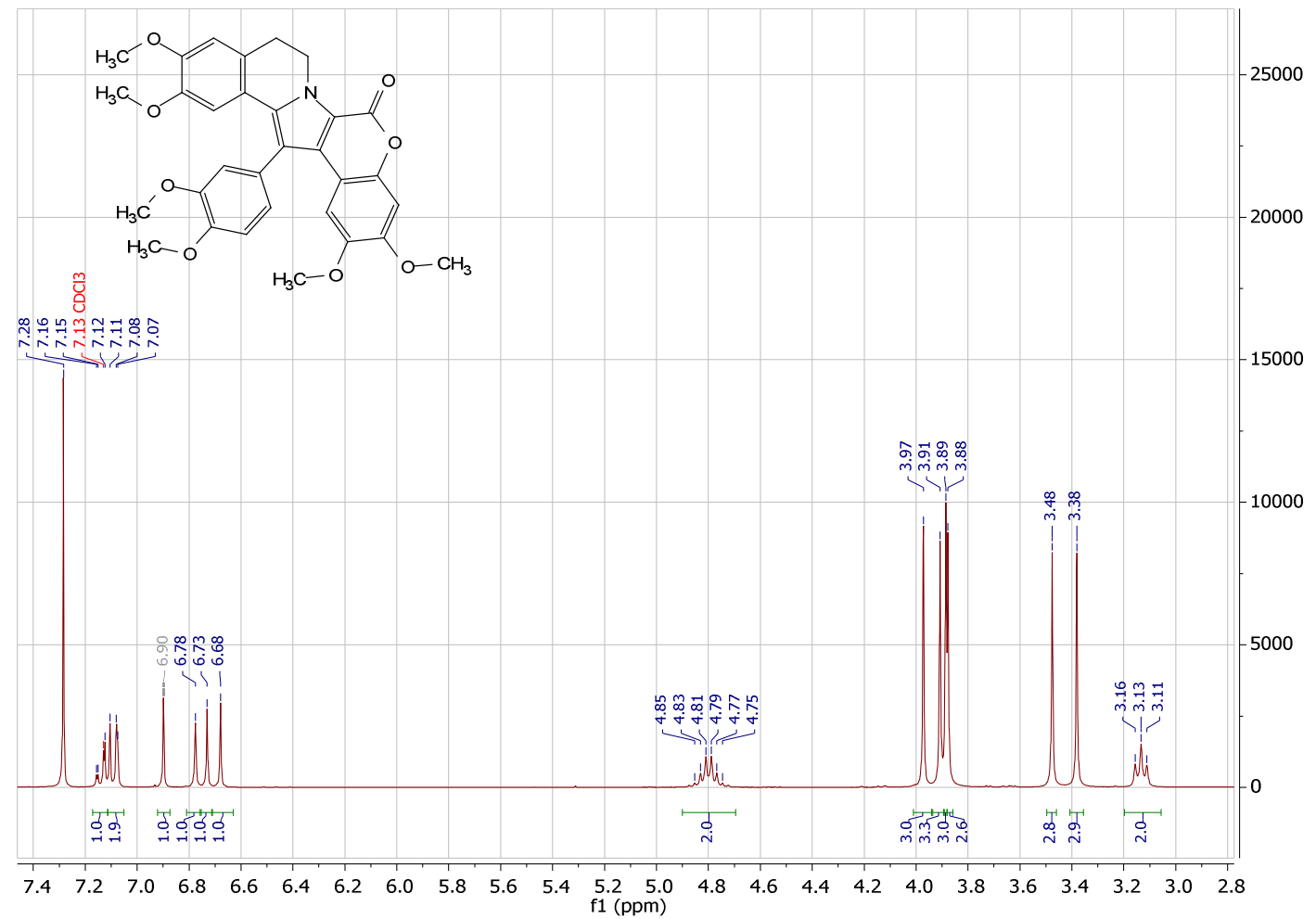

7(b) ${ }^{13} \mathrm{C}\left\{{ }^{1} \mathrm{H}\right\}$ NMR spectrum of lamellarin $\mathrm{G}$ trimethyl ether (3) $\left(75 \mathrm{MHz}, \mathrm{CDCl}_{3}\right)$

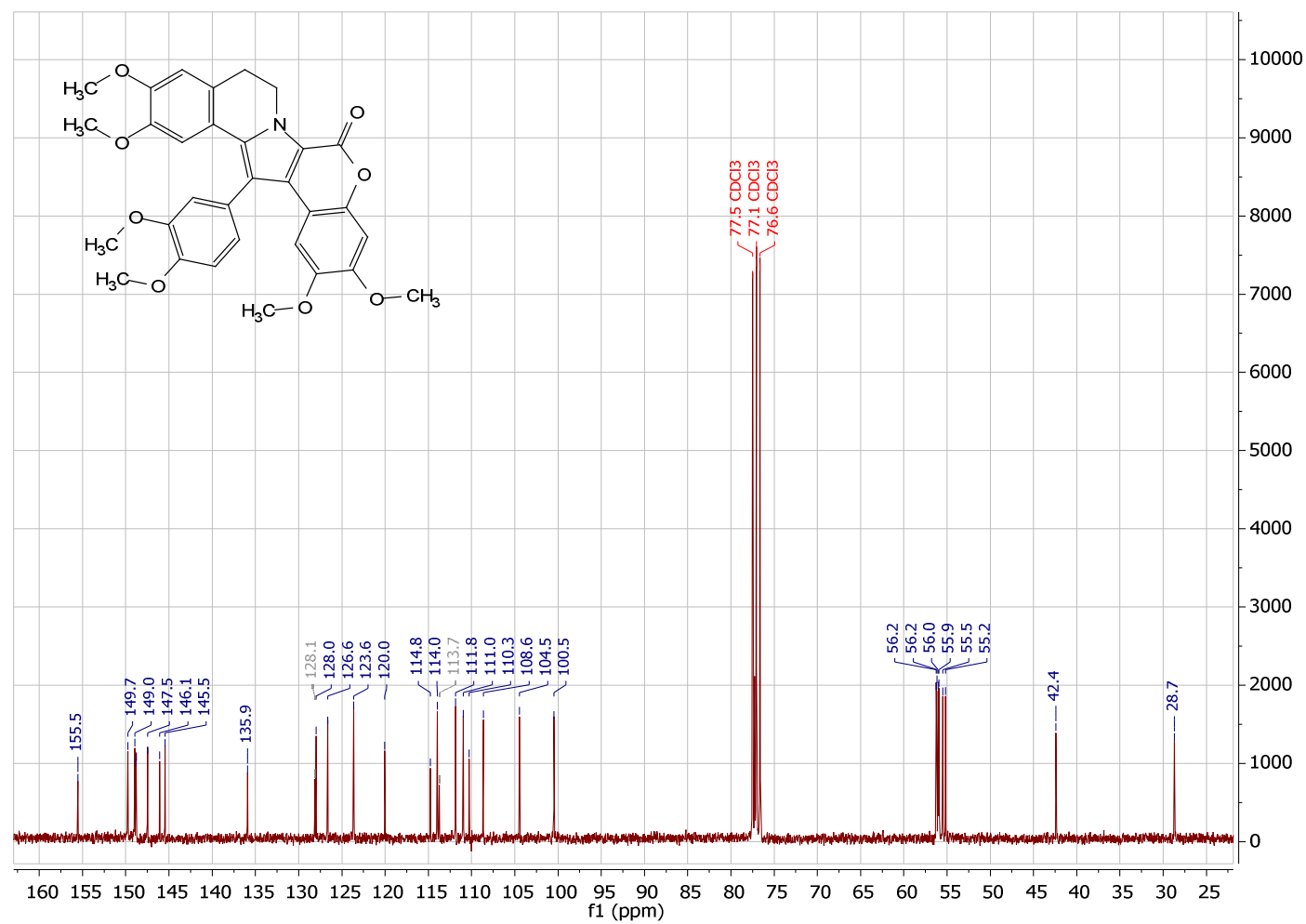

